\title{
Failure After Laparoscopic Pyeloplasty: Prevention and Management
}

\author{
Hung-Jui Tan, M.D., Zaojun Ye, M.S., William W. Roberts, M.D., and J. Stuart Wolf, Jr., M.D., FACS
}

\begin{abstract}
Background and Purpose: Because of the high success of laparoscopic pyeloplasty (LP) for ureteropelvic junction obstruction, strategies for managing failures are less well described. We report our experience with persistent or recurrent obstruction after LP.

Patients and Methods: We reviewed 128 patients who were treated with LP at our institution from 1996 through 2008. Success was defined as objective resolution of obstruction by renal scintigraphy, Whitaker testing, or direct visualization. We extracted data by chart review regarding patient demographics, medical history, operative technique, and salvage treatments. We then assessed for association between patient characteristics and treatment failure.

Results: Overall, 102 patients had sufficient follow-up, of which $84(82 \%)$ were successes. Of 18 failures, median time to failure was 2.5 months ( $0.5-88 \mathrm{mos})$. Of 10 failures managed endoscopically, 7 were salvaged. One of two patients treated conservatively ultimately had resolution while six patients needed simple nephrectomy. Overall, $8(44 \%)$ were salvageable with median follow-up of 19 months (4-58 mos). Patients with failure were more likely to have diabetes mellitus, longer length of stay, higher American Society of Anesthesiologists (ASA) score, a stent placed at the time of pyeloplasty, or ureteral stent malfunction $(P<0.05)$. Patients with failure despite salvage were more likely to have stent malfunction or body mass index $>30 \mathrm{~kg} / \mathrm{m}^{2}(P<0.05)$. Adjusting for the above factors, stent placement at time of surgery and ASA score $>2$ were associated with failure $(P<0.05)$ while periureteral fibrosis trended toward a significant association $(P=0.061)$.

Conclusion: Nearly half of failures after LP are salvageable, many with endoscopic management.
\end{abstract}

\section{Introduction}

L APAROSCOPIC PYELOPLASTY (LP) for ureteropelvic junction obstruction (UPJO) was first described in 1993. ${ }^{1}$ Although technically challenging, LP is associated with high success rates, ranging from $84 \%$ to $98 \% .^{2-5} \mathrm{LP}$ achieves better success rates than endopyelotomy $(\mathrm{EP})$ and comparable results to open pyeloplasty (OP). ${ }^{3,6}$ More recently, robot-assisted LP has gained ground with outcomes equivalent to those of conventional LP. ${ }^{7}$ Given the benefits of easier convalescence, decreased pain, and smaller incisions, ${ }^{6}$ LP (both conventional and robot-assisted) is now considered the standard of care for UPJO at many institutions. ${ }^{4,8}$

Despite the high success rates, some patients have persistent or recurrent UPJO. Various strategies have been used in these situations: Observation, ureteral stent placement, or percutaneous nephrostomy, EP, repeated LP, OP, or nephrectomy. While secondary procedures have been reported in some series, $3,7,9$ data available on salvage strategies and subsequent outcomes are lacking. LP and EP have been both described as salvage therapy for failed pyeloplasty with good results, but these have been primarily after open surgery. ${ }^{10-12}$ Furthermore, potentially modifiable risk factors that are associated with recurrent or persistent obstruction after primary and secondary repair have not been well described.

To gain better insight on the salvage setting, we analyzed our series of patients who were treated with LP to determine failure and success rates. We describe our management strategies for salvage therapy and report our overall outcomes. We also attempt to identify potentially modifiable risk factors associated with persistent or recurrent obstruction that may help urologists minimize failures after laparoscopic repair.

\section{Patients and Methods}

\section{Data source and subjects}

Institutional Review Board approval was obtained to perform a retrospective review of patients who were undergoing LP at the University of Michigan. We identified 128 patients from August 1996 through September 2008. In general, UPJO was diagnosed by diuretic renal scan (DRS) and then

Department of Urology, Division of Endourology and Stone Disease, University of Michigan, Ann Arbor, Michigan. 
confirmed by retrograde pyelography at the time of LP. In four patients, diagnosis was confirmed with Whitaker testing, in three patients for equivocal DRS findings and in 1 because a nephrostomy tube was already in place. A ureteral stent was placed cystoscopically either before LP or at the time of surgery. All LPs were performed transperitoneally, as described previously. ${ }^{13}$ A closed suction drain was placed and removed before discharge unless a urine leak was suspected. The ureteral stent was routinely removed between 2 and 6 weeks postoperatively followed by DRS obtained at 3 weeks, 3 months (if the 3-week result was equivocal), 1 year after stent removal, and variably thereafter. Patients lost to follow-up after stent removal (10) or who opted for local follow-up (14) were excluded. There were two intraoperative conversions to $\mathrm{OP}$, and these patients were excluded from the analysis.

\section{Primary outcome}

We assessed our patients for success or failure both before and after salvage attempt. We defined success as DRS with $T_{1 / 2}$ $\leq 20$ minutes, accompanied by improvement in symptoms. In cases where the DRS result was either equivocal or discordant with the clinical picture (ie, elevated $T_{1 / 2}$ with resolution of symptoms or vice versa), success was defined by negative Whitaker testing (pressure gradient $<20 \mathrm{~cm} \mathrm{H}_{2} \mathrm{O}$ ) or patency of the UPJ on direct ureteroscopic visualization (except in three patients described in the Results section). Remaining cases were deemed failures. Rates of success, success including salvage, failure, and failure despite salvage were calculated. The numerators were number of successes or failures before and after salvage attempt. The denominator was number of patients receiving LP with adequate follow-up, as described above.

\section{Statistical analysis}

A detailed chart review was performed of each case. We extracted data on patient demographics, primary surgeon, surgeon experience, body mass index (BMI), infection, urolithiasis history, surgical history, DRS and radiographic findings, need for urinary diversion, and previous treatment for UPJO. Charlson comorbidity index (CCI) was calculated for each patient. ${ }^{14}$ Intraoperative factors, including American Society of Anesthesiologists (ASA) score, estimated blood loss, operative time, preoperative stent $v s$ stent placed at time of surgery, type of repair, crossing vessel, and periureteral fibrosis, were also recorded. Finally, we assessed patients for postoperative complications (eg, any, urine leak, stent malfunction), duration of follow-up, and management of failures (ie, conservative, endoscopic [eg, balloon dilation, EP], LP, $\mathrm{OP}$, and nephrectomy).

Failures and failures despite salvage were compared with successes with bivariable analyses using parametric or nonparametric testing as appropriate. We then fit a multivariable logistic regression model with potentially modifiable factors identified a priori or through bivariable analyses. For all statistical inferences, we performed two-sided significance testing and set a type I error rate at 0.05 , using the SAS system (v9.2, SAS Institute, Cary, NC).

\section{Results}

Of 128 cases, 102 were available for review. Overall, we identified $84(82 \%)$ successes and $18(18 \%)$ failures. This in- cludes three patients (including one patient who underwent bilateral LPs for horseshoe kidney) we considered to have successful outcomes despite abnormal DRS findings because of complete resolution of pain along with additional evidence of functional improvement (ie, significant reduction in serum creatinine level in two patients and complete resolution of hydronephrosis in the other). Conversely, there were three patients we considered to be failures because they needed an intervention for persistent symptoms despite a DRS $T_{1 / 2} \leq 20$ minutes. Of the 18 failures, median time to failure was 2.5 months $(0.5-88 \mathrm{mos})$. Five patients presented more than 1 year post-LP while 13 patients presented within the first year of surgery. Of these patients, 11 demonstrated evidence of obstruction within the first 3 months postoperatively.

Management of failed LP is detailed in Figure 1. Two patients opted for conservative management, one of whom experienced spontaneous resolution. Seven of 10 patients had successful salvage endoscopically. Of the three patients in whom EP failed, two patients underwent salvage OP: One had persistent obstruction while the other had initial resolution before recurrent obstruction developed 4 years later. Six patients needed simple nephrectomy. These patients demonstrated severe symptoms because of their UPJO $(n=5)$, significant obstruction (DRS $\mathrm{T}_{1 / 2}>99 \mathrm{~min}, \mathrm{n}=2$ ), and/or poorly functioning kidney (post-LP DRS differential $<5 \%$, $\mathrm{n}=3$ ), and were thought to have a significantly low probability of successful salvage. Overall, salvage occurred in 8 (44\%) patients (median follow-up postsalvage of 19 months [4-58 months]), resulting in 92 (90\%) successes including salvage and $10(10 \%)$ failures despite salvage.

The results of our bivariable analyses are reported in Table 1. Comparing successes with both failures and failures despite salvage, we found no significant differences in age, sex, CCI, previous urinary diversion, previous EP, type of repair, periureteral fibrosis, or crossing vessel. Although not included in Table 1, we also found no significant association between failure and side of UPJO, surgical history, primary surgeon, and surgeon experience (all $P$ values $>0.40$ ). Patients who experienced failure were more likely to have diabetes mellitus, a longer length of stay, higher ASA score, a stent placed at time of pyeloplasty, and ureteral stent malfunction $(P<0.05)$. Patients who experienced failure despite salvage were more likely to have stent malfunction or BMI $>30 \mathrm{~kg} / \mathrm{m}^{2}$ $(P<0.05)$. While the frequency of urine leaks and complications overall were similar, patients in whom LP failed were more likely to experience a stent malfunction. Notably, seven of eight patients with stent malfunction returned to the operating room for stent adjustment, change, or removal.

As described in Table 2, stent placement at the time of surgery and ASA score $>2$ were both associated with failure on our multivariable analysis $(P<0.05)$. While neither diabetes mellitus nor BMI $>30 \mathrm{~kg} / \mathrm{m}^{2}$ were found to be associated with failure, periureteral fibrosis trended toward a significant association with persistent or recurrent UPJ obstruction $(P=0.061)$. In contrast, there was no association with these factors and failure despite salvage after adjusting for factors identified a priori or through bivariable analyses (Table 2).

\section{Discussion}

At our institution, LP is associated with an $82 \%$ success rate. Nearly half of our failures were salvageable, most with 


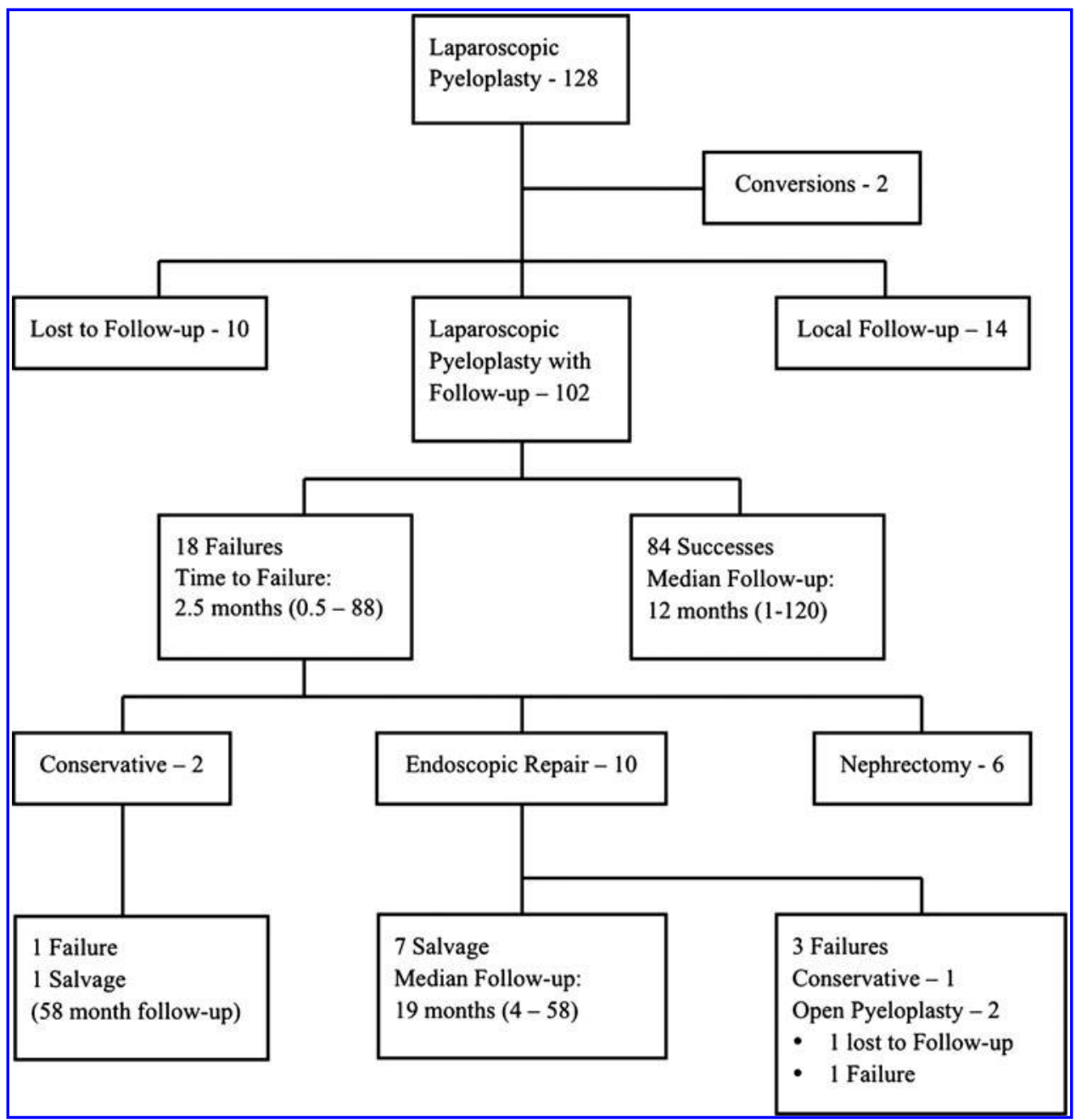

FIG. 1. Management of recurrent or persistent obstruction after laparoscopic pyeloplasty at the University of Michigan, including salvage treatments attempted and outcomes.

conservative management or endoscopic repair. Salvage OP was attempted in two with mixed results. One-third of patients who experienced failure received a simple nephrectomy, many of whom had been counseled about simple nephrectomy for poorly functioning kidney but elected to proceed with an attempted reconstruction. When comparing successes with failures, patients who had failure were more likely to have diabetes mellitus, longer length of stay, higher ASA score, stent placement at time of pyeloplasty, or ureteral stent malfunction. Patients with failure despite salvage were more likely to have a stent malfunction or body mass index $>30 \mathrm{~kg} / \mathrm{m}^{2}$. On multivariable analysis, stent placement at time of pyeloplasty and ASA score $>2$ were associated with failure while periureteral fibrosis trended toward significance.

Our success rate is slightly lower than previously published series. ${ }^{2-4,7,15}$ We did exclude 24 patients who were followed locally or lost to follow-up. Because it is likely that these patients would re-present or be rereferred if clinical evidence of recurrent UPJO developed, our true success rate may be higher. In addition, success rates may, in fact, be lower than previously thought. As seen in our series, many failures occur beyond 1 year after surgery, necessitating the need for longer follow-up. 5,13,16 Moreover, success rates vary according to definition, ${ }^{17}$ and standardized definitions of success after repair of UPJO may allow for better comparisons between series.

As demonstrated by our series, a variety of treatment strategies can be used for the management of recurrent or persistent obstruction after LP. Our findings appear to be consistent with previously published reports (Table 3). Endoscopic management produces fair results and appears to be the most common surgical technique., ${ }^{3,7,9}$ More recently, there have been a few reports of LP as salvage therapy after an initial LP because of the promising results achieved with LP after previously failed OP. ${ }^{7,9-11}$ Given the likelihood of significant fibrosis and good results noted with OP in secondary obstruction, ${ }^{12,18-20}$ we opt for the open approach when patients experience failure with both LP and EP. While we have had good success with OP for secondary UPJO, our results have been mixed after recurrent or persistent obstruction after LP. Interestingly, conservative management may be an increasingly viable option, especially in patients with minimal symptoms and stable renal function. ${ }^{4,5}$ Overall, many patients can have salvage with conservative management or endoscopic repair after failure of LP. 
Table 1. Bivariable Analyses - Failures and Failures Despite Salvage Compared to Successes and Successes Including Salvage, Respectively

\begin{tabular}{|c|c|c|c|c|c|c|}
\hline & Success (\%) & Failure (\%) & $\mathrm{P}$ value & Success including salvage (\%) & Failure after salvage (\%) & P value \\
\hline No. of patients & 84 & 18 & & 92 & 10 & \\
\hline Age (years) & $35.2 \pm 14.6$ & $36.9 \pm 15.9$ & 0.672 & $35.7 \pm 15.2$ & $33.9 \pm 10.7$ & 0.645 \\
\hline Male & 51.2 & 44.4 & 0.796 & 50.0 & 50.0 & 1.000 \\
\hline $\mathrm{BMI}>30 \mathrm{~kg} / \mathrm{m}^{2}$ & 12.2 & 27.8 & 0.138 & 12.2 & 40.0 & 0.041 \\
\hline Diabetes & 3.6 & 22.2 & 0.017 & 5.4 & 20.0 & 0.139 \\
\hline Infection & 23.8 & 11.1 & 0.348 & 23.9 & 0.0 & 0.113 \\
\hline Stone history & 17.9 & 33.3 & 0.196 & 18.5 & 40.0 & 0.209 \\
\hline CCI 0 & 66.7 & 66.7 & 1.000 & 67.4 & 60.0 & \multirow[t]{3}{*}{0.417} \\
\hline CCI 1 & 14.3 & 16.7 & & 13.0 & 30.0 & \\
\hline CCI 2 or more & 19.1 & 16.7 & & 19.6 & 10.0 & \\
\hline Urinary diversion & 63.1 & 61.1 & 0.874 & 62.0 & 70.0 & 0.617 \\
\hline Previous EP & 20.2 & 22.2 & 1.000 & 19.6 & 30.0 & 0.426 \\
\hline DRS $<35 \%^{a}$ & 28.9 & 25.0 & 1.000 & 25.0 & 50.0 & 0.206 \\
\hline DRS $\mathrm{T}_{1 / 2}>20 \mathrm{~min}^{\mathrm{a}}$ & 75.4 & 75.0 & 1.000 & 75.8 & 71.4 & 1.000 \\
\hline OR time $(\min )^{b}$ & $199.8 \pm 57.7$ & $213.8 \pm 84.3$ & 0.510 & $198.1 \pm 56.2$ & $239.4 \pm 103.6$ & 0.244 \\
\hline LOS (days) ${ }^{b}$ & $2.4 \pm 1.1$ & $3.2 \pm 1.5$ & 0.046 & $2.4 \pm 1.1$ & $3.4 \pm 1.8$ & 0.124 \\
\hline $\mathrm{EBL}(\mathrm{mL})^{\mathrm{b}}$ & $73.1 \pm 60.2$ & $73.2 \pm 30.0$ & 0.992 & $72.7 \pm 58.1$ & $79.5 \pm 33.8$ & 0.666 \\
\hline ASA 1 & 54.8 & 38.9 & \multirow[t]{3}{*}{0.045} & 54.4 & 30.0 & \multirow[t]{3}{*}{0.074} \\
\hline ASA 2 & 42.9 & 44.4 & & 42.4 & 50.0 & \\
\hline ASA $3+$ & 2.4 & 16.7 & & 3.3 & 20.0 & \\
\hline Stent at time of LP & 61.9 & 88.9 & 0.030 & 64.1 & 90.0 & 0.159 \\
\hline Dismembered & 77.4 & 72.2 & 0.640 & 77.2 & 70.0 & 0.612 \\
\hline Simple ND & 21.4 & 22.2 & 1.000 & 21.7 & 20.0 & 1.000 \\
\hline Complex repair & 1.2 & 5.6 & 0.323 & 1.1 & 10.0 & 0.187 \\
\hline Crossing vessel & 63.1 & 55.6 & 0.599 & 64.1 & 40.0 & 0.176 \\
\hline Fibrosis & 25.0 & 44.4 & 0.147 & 27.2 & 40.0 & 0.464 \\
\hline Any complication & 31.0 & 50.0 & 0.122 & 31.5 & 60.0 & 0.072 \\
\hline Urine leak & 17.9 & 16.7 & 1.000 & 17.4 & 20.0 & 1.000 \\
\hline Stent complication & 4.8 & 22.2 & 0.031 & 5.4 & 30.0 & 0.030 \\
\hline
\end{tabular}

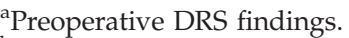

${ }^{\mathrm{b}}$ Continuous variables are presented as mean \pm standard deviation.

$\mathrm{BMI}=$ body mass index; $\mathrm{CCI}=$ Charlson comorbidity index; $\mathrm{EP}=$ endopyelotomy; $\mathrm{DRS}=$ diuretic renal scan; $\mathrm{OR}=\mathrm{operating}$ room; $\mathrm{LOS}=$ length of stay; $\mathrm{EBL}=$ estimated blood loss; $\mathrm{ASA}=$ American Society of Anesthesiologists; $\mathrm{LP}=$ laparoscopic pyeloplasty; ND= nondismembered repair.

Given the variable salvage rates and need for subsequent procedures, the best management of failure may be prevention. To date, risk factors for persistent or recurrent obstruction after LP have not been well defined. Hydronephrosis, poor renal function, and crossing vessel have been identified as risk factors for EP while ASA score and BMI have been associated with complications of urologic laparoscopy. ${ }^{18,21-23}$ Based on our analysis, higher ASA score and obesity may be specific risk factors for failure after LP. Perhaps more interesting is the potential relationship between diabetes mellitus and failure after LP. It has been hypothesized that diabetes-related microvascular disease negatively impacts a healing anastomosis because diabetes mellitus has been linked to poorer outcomes in procedures that involve reconstruction of the urinary tract. ${ }^{24-26}$ Unlike ASA score and obesity, diabetes may be more modifiable in

Table 2. Multivariable Analysis -Failure And Failure Despite Salvage

\begin{tabular}{lcccc}
\hline & \multicolumn{2}{c}{ Failure } & & Failure after Salvage \\
\cline { 2 - 3 } Variable & Odds ratio $(95 \% \mathrm{CI})$ & P value & Odds ratio $(95 \%$ CI) & $\mathrm{P}$ value \\
\hline ASA 2 vs. 1 & $0.99(0.27-3.58)$ & 0.988 & $1.57(0.31-8.05)$ \\
ASA 3+ vs. 1 & $20.92(1.10-399.36)$ & 0.043 & $12.72(0.56-286.89)$ & 0.585 \\
BMI > 30 kg/m & $1.01(0.20-5.20)$ & 0.989 & $2.16(0.36-12.84)$ & 0.110 \\
Diabetes mellitus & $3.61(0.53-24.48)$ & 0.189 & $1.94(0.12-10.41)$ & $0.43-8.76)$ \\
Fibrosis & $3.19(0.95-10.74)$ & 0.061 & $8.28(0.66-104.14)$ & 0.391 \\
Stent at time of LP & $12.50(1.38-113.00)$ & 0.025 & 0.102 \\
\hline
\end{tabular}

$\mathrm{CI}=$ confidence interval; $\mathrm{ASA}=$ American Society of Anesthesiologists; $\mathrm{BMI}=$ body mass index; $\mathrm{LP}=$ laparoscopic pyeloplasty. 
Table 3. Review of Management of Failed Laparoscopic Pyeloplasty

\begin{tabular}{|c|c|c|c|c|c|c|c|}
\hline Series & Failures & Conservative management & Endoscopic repair & $L P$ & $O P$ & Simple nephrectomy & Salvage \\
\hline Maynes $^{5}$ & 7 & 3 & 1 & 0 & 0 & 1 & - \\
\hline Moon $^{4}$ & 6 & 2 & 2 & 0 & 1 & 0 & - \\
\hline Mufarrij ${ }^{7}$ & 6 & 0 & 2 & 2 & 0 & 2 & 4 \\
\hline Rassweiler $^{3}$ & 8 & 0 & 4 & 0 & 3 & 1 & - \\
\hline Szydelko $^{15}$ & 3 & 1 & 1 & 0 & 2 & 0 & 2 \\
\hline Varkarakis $^{9}$ & 10 & 0 & 10 & 1 & 2 & 0 & 10 \\
\hline
\end{tabular}

$\mathrm{LP}=$ laparoscopic pyeloplasty; $\mathrm{OP}=$ open pyeloplasty.

terms of preoperative and perioperative management. General guidelines have been developed regarding the management of hyperglycemia and diabetes around the time of surgery. ${ }^{27}$

Repair of UPJO may be more technically challenging in the salvage setting because of fibrosis and inflammation. While there are multiple etiologies for fibrosis (eg, infection, previous instrumentation, previous abdominal surgery), significant fibrosis has been observed during both laparoscopic and open surgery after previous EP. ${ }^{11,18-20}$ While we did not identify EP as a risk factor for failure, lower success rates have been reported with LP for secondary UPJO, ${ }^{2,5}$ and preliminary findings from a larger multi-institutional study have identified previous EP as a potential risk factor for failure after robot-assisted and conventional LP. ${ }^{28}$ Steps to minimize fibrosis, including altering our practice patterns with respect to $\mathrm{EP}$, may decrease the occurrence of failures after pyeloplasty.

Our findings must be considered in the context of several limitations. As a retrospective analysis, we are vulnerable to potential confounders. In fact, we found stent placement at the time of pyeloplasty to be associated with failure even though the disadvantages of a preplaced stent (eg, decompressed collecting system, risk of displacement or damage, inflammation, edema) have been well described. ${ }^{2,29}$ Given the association between postoperative stent malfunction and failure in our series, stent placement practices may play a larger role in affecting the outcome after LP and may deserve further study. It is just as plausible, however, that patients with a stent in place may be predisposed toward success for other reasons. For example, they may be more symptomatic or have more severe obstruction.

Next, we may be underpowered to identify certain factors associated with failure after LP despite comparable size to other series. A larger, multi-institutional study as mentioned above was able to identify EP as a risk factor, and it may be more generalizable given the larger cohort size. Our more complete dataset, however, enables us to examine other clinical factors more precisely. Despite this advantage, we were still unable to incorporate specific renal scan parameters or degree of hydronephrosis in our analyses because of variations in interpretation of imaging studies; many of these were from outside institutions. These objective measures may provide additional information in future assessments of UPJO and its surgical management.

These limitations notwithstanding, our findings have potential implications on the management of UPJO. The majority of practicing urologists currently use EP as initial therapy for primary UPJO despite lower rates of success when compared with pyeloplasty. ${ }^{3,5,8,16}$ Given the association of failure with fibrosis and potentially EP, EP should be used conservatively in the primary setting and may be better used as salvage therapy for recurrent UPJO. ${ }^{3,30}$ Presence of a crossing vessel, marked hydronephrosis, and previous failed EP appear to affect treatment selection for UPJO among practicing urologists. ${ }^{8}$ Based on our findings, ASA score, BMI, and diabetes mellitus should also be considered. Given the good success rates of LP, incorporating these factors into the decision-making may further minimize an already low failure rate after LP. If failure should occur, conservative options such as observation or endoscopic management are likely to yield good results.

\section{Conclusion}

LP is now the standard of care for UPJO at many institutions. Although uncommon, failures can occur but are often manageable with conservative or minimally invasive techniques. Stent placement at time of LP, fibrosis, ASA score, obesity, and diabetes mellitus may impact the outcome of LP. Consideration of these factors during decision-making may help minimize the occurrence of failures after LP.

\section{Disclosure Statement}

William W. Roberts has royalty, equity, and consulting interests in HistoSonics, Inc. No competing financial interests exist for the remaining authors.

\section{References}

1. Schuessler WW, Grune MT, Tecuanhuey LV, Preminger GM. Laparoscopic dismembered pyeloplasty. J Urol 1993; 150:1795-1799.

2. Jarrett TW, Chan DY, Charambura TC, et al. Laparoscopic pyeloplasty: The first 100 cases. I Urol 2002;167:1253-1256.

3. Rassweiler JJ, Subotic S, Feist-Schwenk M, et al. Minimally invasive treatment of ureteropelvic junction obstruction: Long-term experience with an algorithm for laser endopyelotomy and laparoscopic retroperitoneal pyeloplasty. J Urol 2007;177:1000-1005.

4. Moon DA, El-Shazly MA, Chang CM, et al. Laparoscopic pyeloplasty: Evolution of a new gold standard. Urology 2006;67:932-936.

5. Maynes LJ, Levin BM, Webster TM, et al. Measuring the true success of laparoscopic pyeloplasty. J Endourol. 2008;22: 1193-1198.

6. Brooks JD, Kavoussi LR, Preminger GM, et al. Comparison of open and endourologic approaches to the obstructed ureteropelvic junction. Urology 1995;46:791-795.

7. Mufarrij PW, Woods M, Shah OD, et al. Robotic dismembered pyeloplasty: A 6-year, multi-institutional experience. J Urol 2008;180:1391-1396. 
8. Tan BJ, Rastinehad AR, Marcovich R, et al. Trends in ureteropelvic junction obstruction management among urologists in the United States. Urology 2005;65:260-264.

9. Varkarakis IM, Bhayani SB, Allaf ME, et al. Management of secondary ureteropelvic junction obstruction after failed primary laparoscopic pyeloplasty. J Urol 2004;172: 180-182.

10. Shapiro EY, Cho JS, Srinivasan A, et al. Long-term follow-up for salvage laparoscopic pyeloplasty after failed open pyeloplasty. Urology 2009;73:115-118.

11. Sundaram CP, Grubb RL III, Rehman J, et al. Laparoscopic pyeloplasty for secondary ureteropelvic junction obstruction. J Urol 2003;169:2037-2040.

12. Ng CS, Yost AJ, Streem SB. Management of failed primary intervention for ureteropelvic junction obstruction: 12-year, single-center experience. Urology 2003;61:291-296.

13. Madi R, Roberts WW, Wolf JS Jr. Late failures after laparoscopic pyeloplasty. Urology 2008;71:677-681.

14. Charlson ME, Pompei P, Ales KL, MacKenzie CR. A new method of classifying prognostic comorbidity in longitudinal studies: Development and validation. I Chronic Dis 1987;40:373-383.

15. Szydelko T, Kasprzak J, Apoznanski W, Zdrojowy R. Laparoscopic anterior $\mathrm{y}-\mathrm{v}$ pyeloplasty: A valuable treatment option in patients with small or intrarenal pelvis. J Laparoendosc Adv Surg Tech A 2010;20:627-630.

16. Dimarco DS, Gettman MT, McGee SM, et al. Long-term success of antegrade endopyelotomy compared with pyeloplasty at a single institution. J Endourol 2006;20:707712.

17. Pouliot F, Lebel MH, Audet JF, Dujardin T Determination of success by objective scintigraphic criteria after laparoscopic pyeloplasty. I Endourol 2010;24:299-304.

18. Kavoussi LR, Albala DM, Clayman R. Outcome of secondary open surgical procedure in patients who failed primary endopyelotomy. Br J Urol 1993;72:157-160.

19. Gupta M, Tuncay OL, Smith AD. Open surgical exploration after failed endopyelotomy: A 12-year perspective. J Urol 1997;157:1613-1619.

20. Motola JA, Fried R, Badlani GH, Smith AD. Failed endopyelotomy: Implications for future surgery on the ureteropelvic junction. I Urol 1993;150:821-823.

21. Van Cangh PJ, Wilmart JF, Opsomer RJ, et al. Long-term results and late recurrence after endoureteropyelotomy: A critical analysis of prognostic factors. I Urol 1994;151: 934-937.

22. Permpongkosol S, Link RE, Su LM, et al. Complications of 2,775 urological laparoscopic procedures: 1993 to 2005. I Urol 2007;177:580-585.
23. Gabr AH, Gdor Y, Strope SA, et al. Patient and pathologic correlates with perioperative and long-term outcomes of laparoscopic radical nephrectomy. Urology 2009;74:635-640.

24. Borboroglu PG, Sands JP, Roberts JL, Amling CL. Risk factors for vesicourethral anastomotic stricture after radical prostatectomy. Urology 2000;56:96-100.

25. Breyer BN, McAninch JW, Whitson JM, et al. Multivariate analysis of risk factors for long-term urethroplasty outcome. J Urol 2010;183:613-617.

26. Nichols WK, Krause AH, Donegan WL. Urinary fistulas after ureteral diversion. Am J Surg 1972;124:311-316.

27. Moghissi ES, Korytkowski MT, DiNardo M, et al. American Association of Clinical Endocrinologists and American Diabetes Association consensus statement on inpatient glycemic control. Diabetes Care 2009;32:1119-1131.

28. Lucas S, Leveillee R, Bird V , et al. Multi-institutional comparison of outcomes of robot-assisted and laparoscopic pyeloplasty. Abstract presented at: 84th Annual North Central Section of the American Urological Association; Chicago, IL; September 30, 2010.

29. Mandhani A, Goel S, Bhandari M. Is antegrade stenting superior to retrograde stenting in laparoscopic pyeloplasty? J Urol 2004;171:1440-1442.

30. Yong D, Albala DM. Endopyelotomy in the age of laparoscopic and robotic-assisted pyeloplasty. Curr Urol Rep 2010;11:74-79.

Address correspondence to:

J. Stuart Wolf, Jr., M.D., FACS Department of Urology University of Michigan

1500 East Medical Center Drive 3875 Taubman Center Ann Arbor, MI 48109

E-mail:wolfs@med.umich.edu

\begin{aligned} & \multicolumn{1}{c|}{ Abbreviations Used } \\ & $\mathrm{ASA}=$ American Society of Anesthesiologists \\ & $\mathrm{BMI}=$ body mass index \\ & $\mathrm{CCI}=$ Charlson comorbidity index \\ & $\mathrm{DRA}=$ diuretic renal scan \\ & $\mathrm{EP}=$ endopyelotomy \\ & $\mathrm{LP}=$ laoparoscopic pyeloplasty \\ & $\mathrm{OP}=$ open pyeloplasty \\ & $\mathrm{UPJO}=$ ureteropelvic junction obstruction \end{aligned}

